\title{
Videos in learning in higher education: assessing perceptions and attitudes of students at the University of Ghana
}

\author{
Richard Boateng, Sheena Lovia Boateng, Raphael Baffour Awuah, Eric Ansong* and Augustus Barnnet Anderson
}

\author{
* Correspondence: \\ eansong003@st.ug.edu.gh \\ University of Ghana, Accra, Ghana
}

\begin{abstract}
Video is one of the most diversified and distinct virtual learning mediums that capture and present information and offer a sensory learning environment, which enables learners to understand more and retain information better. This study sought to assess the perceptions and attitudes of students at the University of Ghana towards the use of videos as a medium for teaching and learning. Qualitative data was collected using semi structured interviews. Participation was entirely voluntary and was conditional, based on students' verbal consent. A convenience sample of 20 students responded to a request to participate, and data was analyzed using content analysis. Students perceived videos in general as being of some benefit to their learning activities. Overall, comments on videos as a medium of teaching and learning were positive. However, students had negative perceptions about the nature of the videos they watched. Almost all the participants indicated that they had issues with the content and the format of all the videos they watched. But, majority of the participants perceived that the videos they watched enhanced their learning outcomes and improved their learning approach. Therefore, learning outcomes of students and instructors should be dependent on the manner in which videos are used, as part of the overall academic process. This study is one of the first articles to explore in detail, students' perceptions and attitudes towards video based teaching in Ghana, and provides interesting insights with regard to the concept and its application in tertiary institutions.
\end{abstract}

Keywords: Video supported learning, E-learning, Higher learning institutions, Developing countries

\section{Introduction}

In most of sub-Saharan Africa (SSA), the field of education is confronted with a serious challenge of keeping pace with the rapid technological changes that the society is undergoing; and adapting such technologies to classroom teaching and learning. The main challenge has been with the inability to develop the best teaching and learning environment, on the basis of video orientated communication (Luppicini 2003; Jonassen 2000; O'Flaherty and Phillips 2015). Video, one of the most diversified and distinct virtual learning mediums captures and presents information; and offers a sensory learning environment that allows learners to understand more and retain information better (Fern et al. 2011; Syed 2001). The value of videos also lies in the ways they are put to use in real life teaching and learning situations

(C) 2016 Boateng et al. Open Access This article is distributed under the terms of the Creative Commons Attribution 4.0 International License (http://creativecommons.org/licenses/by/4.0/), which permits unrestricted use, distribution, and reproduction in any medium, provided you give appropriate credit to the original author(s) and the source, provide a link to the Creative Commons license, and indicate if changes were made. 
(Karppinen 2005). Learning activities that students perform with videos play a critical role in learning outcomes (Karppinen 2005; Boyle 1997).

Many educators and researchers have promoted video as an important tool for providing the context or starting point for learning. Videos provide good illustrations of digitized images, text and sound that can be uploaded to a shared virtual community (Goldman 2007). Videos can also be interlinked with slides, supporting texts, resource links, discussion boards, chat platforms among others, as part of a virtual learning environment (Karppinen 2005). The use of videos is ideal for providing feedback, conducting assessments and also improving the quality of mentoring. Learning, through the use of video technology has also proven to have an impact on the understanding of dynamic events (Duffy and Cunningham 1996). For example, for language learners, video offers the possibility for them to witness the dynamics of interaction. Nonetheless, these important gains have been hardly felt in most educational institutions in Sub-Sahara Africa.

On the other hand, it has been argued that videos may show only part of the story in classroom teaching, which is subject to personal bias and interpretation (Goldman 2007). Additionally, Clark (1994) noted that assessing the effectiveness of video as an instrumental medium can be complicated and to an extent contentious. Another challenge of video as an instructional medium is the cost burden, especially to resource constrained institutions. The production of high quality videos is expensive; and most tertiary institutions in Sub-Saharan Africa and other developing countries may not be able to sustain this form of teaching without reliance on external funding.

Over the last two decades, video based instruction, as an educational medium has gained acceptance as an alternative and a supplement to traditional classroom instruction (Alavi et al. 1997; Rahm and Reed 1997). Thus, the use of videos as an instructional means of teaching is gradually becoming very popular in Sub-Sahara African countries like Ghana, especially among tertiary institutions.

In the last decade, universities in Ghana have made progress in developing Information Communication Technology (ICT) infrastructure. However, there has been a gap between improving ICT infrastructure and the integration of technology, particularly videos into the teaching and learning process (Awidi 2008). It is worth noting that the traditional classroom mode of teaching still remains the most common and widely used in Ghanaian universities. But one avenue for most (public) universities in Ghana to explore and expand video-based teaching and learning is in the development of distance education programs. The modules in this program allow students to work at their own pace and time, rather than scheduling their lives around a set classroom time; making the use of videos in this program an important element. Another avenue to promote the use of video in teaching and learning is integrating videos and making it a necessary component for courses that are practical in nature and content.

Unfortunately, most (public) universities in Ghana have not taken advantage of these opportunities. The underlying factors of this phenomenon have been the organizational or institutional capacity to sustain video as a means of instruction and the cultural resistance associated with change. In order to address these issues, the current study seeks to explore the perceptions and attitudes of students at the University of Ghana 
towards the use of video as a medium of instruction. The study specifically addresses three research questions:

1. How does the use of videos influence students' perceptions of learning and engagement?

2. In what ways does video influence students' attitudes towards learning?

3. How do students use videos to enhance learning and improve their performance?

The theoretical framework for this study emphasizes the importance of situating video as an instructional medium in a more meaningful and problem-solving context. This will provide a better teaching and learning environment for students and therefore function as an important tool for improved student learning outcomes and performance. The subsequent sections of the paper contain a review of related literature and the methods adopted to explore the perceptions and attitudes of video based teaching. The analysis approach is also explained. The results and its implications are also discussed.

\section{Literature review}

In a review of studies on video based teaching, Cennamo (1993) identified three key factors that could potentially influence teaching and learning. Firstly, the content of the videos and the topic of discussion; secondly, the perception of the usefulness of video by the (intended) user; and thirdly, the purpose for which the video is used. It has been suggested that information presented in a visual format is more memorable; and the combination of audio and visual information can better aid comprehension and retention (Kozma 1991; Baggett 1984). A number of studies have opined that the combination of audio and visual information in material promotes better retention, than those presented through a single information source (Nugent 1982). Additionally, results from studies conducted among university students have suggested that video improves learning outcomes.

For instance, a study of 147 psychology students reported that video was a more effective mode of instruction than text for presenting real-life situations in order to enhance the learner's comprehension, retention and satisfaction (Choi and Johnson 2007). In another study among English and Management students, it was reported that digital video promoted the contextual aspects of learning, as well as the emotional involvement in the entire learning process (South et al. 2008; Hakkarainen et al. 2007).

Furthermore, it has been suggested that video can connect knowledge to relevant tasks, activities, contexts, as well as cultures in which it is used (described as situated cognition) (South et al. 2008; Brown et al. 1989). Video enables learning through reflection, while a face-to-face setting or the traditional classroom setting is characterized by more collaboration and conversation which may be difficult to achieve (South et al. 2008). Lange (2008) also argues that video may also stimulate participation and impact positively on learning processes and outcomes. Prensky (2005) suggests that young students are especially receptive to video content because they find it attractive (or enjoy the use) and this benefits them in one way or 
the other in the learning process. In a survey comparing the use of a range of teaching techniques, including PowerPoint lectures and videos, younger students (and male students) reported greater enjoyment, but not necessarily learning from video; while older students preferred learning more from video lectures (Tang and Austin 2009).

It is worthy to note that the theoretical rationale for the efficacy of video is absent from most studies. However, the Cognitive Theory of Multimedia Learning (Mayer 2009) comes closest to explaining the literature on teaching with videos. This theory has been developed over time; and it views learning as a process of knowledge construction. Mayer argues that multimedia instruction (that is, any teaching material(s) that includes words and pictures or video) leads to better learning outcomes than just using words alone. It has been suggested that multimedia materials accomplish this by assisting the sense-making process through the activation of verbal and visual cognitive processes concurrently (Mayer 2009). The multiple channels of delivery, representation of ideas, and sensory stimulation provided by multimedia results in a higher cognitive activity, enhanced retention and understanding of content (Fee and Budde-Sung 2014; Mayer 2009).

Additionally, Mayer $(2008,2009)$ suggests that to use multimedia effectively, excessive information processing should be minimized; there should also be a simplification of how important information is processed; and a process where new information is organized and integrated to makes sense. However, Mayer's principles for using multimedia materials, essentially ignore the learners' cultural background or language capabilities (Fee and Budde-Sung 2014).

Mayer also argues that, the significance of video is dependent on a range of factors, including the way it is used. For instance, unnecessary video content which is not essential to the core curriculum has the potential to hinder learning by overloading students and distracting energy and attention-also known as extraneous cognitive processing (Mayer 2009). In other words, the overuse of technology may negatively impact (rather than assist) learning and retention. Also, the experiences and preferences of learners, which shape or determine to an extent, how and what is learned is also an important factor to consider in multimedia material. However, a challenge to this is the great diversity that has emerged from the globalization of university campuses. It has been suggested that tertiary education may be one of the most globalized sectors in every world region with students and lecturers more mobile than before (Saltmarsh and Swirski 2010).

Furthermore, the opportunity for simultaneous participation provided by videos eliminates a student's need to compete to be recognized by the lecturer and other students (Strauss 1996; Lynn 2013). As a result, students who are introverts can participate in the teaching and learning process without competing for attention, and students who are extroverts will not have to wait until being recognized to participate in the teaching process or discussions (Strauss 1996; Yellen et al. 1995). Also, the use of video as opposed to the traditional classroom experience reduces the student's social presence; and this reduces the nervousness, apprehension or fear associated with self-presentation (Corston and Colman 1996; Strauss 1996). More broadly, these features of video enhance participation more equally across participants (Strauss 1996; Dede 1990). 


\section{Research methodology}

\section{Class description}

The study was conducted after the second semester of the 2014/15 academic year at the University of Ghana Business School among third year undergraduate students taking a course in Research Methods. The course provided students with an initial overview of the key terms and concepts of conducting research. As such, it was expected that most students could apply these terms and concepts directly to their final project work. Structuring the course in a discussion-based format allowed the students to be co-producers of course content and learning (Lengnick-Hall and Sanders 1997). Video lectures were used throughout the semester in addition to other teaching methods such as traditional lectures, tutorial discussions and prescribed readings. Students were then required to submit questions during the scheduled class period. All the students in this study were regular or full time students.

\section{The lecture videos}

Thirteen sessions of recorded lecture videos were used for this research. The videos were in .mov and .MP4 formats. These formats are deemed to produce high quality videos which can be accessed on most devices. Each session of the video lecture lasted an hour where the course facilitator delivered the lecture using a smart board with some students providing the right ambience for viewers of the videos. In the videos, the students pose questions where the facilitator provides answers. Each video was based on a topic which was part of the semester's syllabus, and was given to the students at least 3 days prior to the scheduled class period.

\section{Research design and sample}

Given the lack of empirical research on this subject area (especially in the Ghanaian context), the study was designed as an inductive exploration. Qualitative data was collected employing the use of semi structured interviews. Participation was entirely voluntary and was based on students giving a verbal consent. It was also made clear to the participants that they could withdraw from the study at any stage without penalty. Also, the participants in this study had assurances of anonymity of responses. No inducements were offered to participants. The data was collected at the end of the 13-week course in Research Methods in which video was used in a range of ways as a structured part of the syllabus. The convenience sample comprised 20 students who responded to a request to participate. Individual interviews were designed to explore students' personal perceptions and attitudes in much greater depth. Interviews lasted between 20-30 min. All interviews were audio recorded and transcribed in full.

\section{Data analysis}

Data was analyzed employing the use of content analysis. Content Analysis is a process of reducing and structuring text by identifying, coding, and classifying it into categories (Patton 2002). The process requires a systematic and objective identification of patterns and characteristics in the data, by labeling and classifying strings of text relating to a single theme. This will commonly result in some aspects of the data 
being quantified (Silverman and Marvasti 2008). The basic units of coding were sentences which ranged in length from few lines to several sentences. Data were analyzed manually.

\section{Findings}

Overall, the students who participated in this study responded to the questions they were asked in detail. The bulk of students' responses related to their perceptions of videos. Attitudes towards video based teaching were on issues around the students' learning approach. The findings are presented in themes with the codes from the analysis being described and appropriate quotes provided.

\section{General perception of videos}

The essence of this theme was to capture the overall perception of students with regard to the use of videos for educational purposes in general, and not necessarily videos that were related to the Research Methods course. The perceptions of students varied; from responses that could be categorized as positive and those that could be described as negative. The positive perceptions included the videos being useful, innovative and convenient, and also adding a new perspective on content. The positive perceptions of videos in general are described below.

Most of the students (one-third of the respondents) regarded the use of videos in general as 'useful' or 'helpful'. Some of the students who indicated this had previously used videos to assist in their learning; before the introduction of videos in the Research Methods class. For example, some students stated this:

"I think it is actually useful. There was this time that I missed class and I had to fall on the video to make up for that missed class. It was like watching a [television] series; and it was good." (Female student, 20 years)

"They [videos] are actually helpful because you get the opportunity to go over the video, over and over again." (Female student, 22 years)

One of the students perceived videos as adding a 'new perspective on content'. He indicated that;

"With the video it is like you are relearning everything. You are able to take notice of something new that you may have missed in class."

(Male student, 23 years)

Some of the students indicated that videos were 'innovative' and 'exciting'. For such students, videos made learning entertaining. Some of the students stated this:

"I am drawn to such things like movies and other clips. So video makes learning fun and exciting." (Female student, 23 years)

"I think it is innovative and exciting compared to the long hours spent in a classroom or sitting behind a book." (Male student, 21 years) 
For some students, videos generally provide a 'better understanding' of topics or issues relating to specific areas. To such students, videos have the advantage of enabling deeper understanding. A student stated this:

"Video helps me to understand whatever is being taught much better."

(Female student, 21 years)

However, for this student, even though videos enhance understanding, it should be complimented with traditional face-to-face learning.

"... without one-on-one learning, you can't achieve much [with videos]."

(Female student, 21 years)

The other positive perceptions of videos stated by the students were that videos were 'mobile' and 'convenient'. According to some of the students, videos make it possible to learn or study on the go or at one's own convenience. Some of the students indicated this:

"It is easy to watch. I can watch it on my phone. I can watch it anywhere."

(Female student, 22 years)

"With videos I can watch again and again in the comfort of my room or home." (Female student, 21 years)

"I think that they are convenient. They make your life as a student easy. You can use these videos at your time even in the absence of the lecturer. It's a convenient thing." (Female student, 21 years)

On the other hand, some students indicated some negative aspects of videos. One of these negative perceptions has to do with issues around access. One student indicated that:

"With videos if you don't have a laptop you can't watch. With the energy crisis in the country too, you would have to go to some place with power before you are able to watch the video lectures." (Female student, 21 years)

Another negative perception the participants indicted is the fact that naturally some students may not be interested or have the enthusiasm to watch videos. Such students indicated that the time and effort used in watching videos can be burdensome and can be replaced by reading to understand one concept or the other. As such, videos in general are not an effective means of teaching. One student stated this:

"I am not particularly enthused about videos in general. When I watch videos I usually fall asleep so I actually don't appreciate it. I would prefer to read than to watch the videos because it is almost the same thing. If I read, I may take a minute to understand but with the videos I have to watch for about 30 minutes to understand a topic or issue." (Female student, 21 years) 


\section{Perceptions of the nature of videos}

This theme captures students' perceptions of the videos they used or experienced during the Research Methods course. These perceptions were on issues around the content and format of the videos. With regard to the content of the videos, the students' perceptions were on issues concerning the duration of the videos, how uninteresting or boring the videos were and the fact that some of the videos were similar in content with the normal class lectures. These perceptions about the content of the videos can be categorized as negative. Some of the students stated the following with regard to the content of the videos.

"The video environment needs not to be a dull environment but should be an interactive environment." (Male student, 23 years)

"My problem was that it was really long. People don't like it when things are very long. So in as much as the lecturers want to explain into detail they should not make the videos very long." (Female student, 20 years)

"What they teach in class is the same as what you have in the videos. So the content is the same as that of the usual class. It is just that you may not be in the video because you were not part of the students who shot the video."

(Female student, 23 years)

Also, the perceptions of students with regard to the nature of the videos had to do with issues concerning the format of the videos they experienced. The students' perceptions about the format of the videos were also negative (just as their perceptions about the content). Students' perceptions on the format of the videos were about the size, as well as issues around editing. More than a third of the students perceived that the size of the videos were too large. They indicated that this made accessing and keeping the videos very difficult since their storage devices had limited capacity. A couple of the students (two participants) also indicated that there were some mistakes in the videos, which could have been omitted but were included the final output.

"...the sizes are very large so it makes it very difficult to get the videos onto your laptop." (Female student, 21 years)

"Sometimes the editing of the videos wasn't done properly because you see some mistakes the lecturer made that should have been edited but are still in the video.

Some of these lapses should be avoided." (Male student, 21 years)

\section{Perceived learning outcomes}

This theme captures the learning outcomes that students perceived they gained from the use of videos. The perceived outcomes were either positive or negative. However, two students indicated that the use of videos did not have any impact on their learning outcomes. With regard to the positive outcomes, students indicated that the videos enabled them to get a better/deeper/easier understanding, assisted them to easily recall and made learning easier or better. More than half of the students perceived that the 
videos they experienced enabled them to have a better/deeper/easier understanding of topics. For example, one student stated this:

"It [videos] makes me understand the topic being taught in detail. For example, during our end of semester examinations, I could not understand some of the PowerPoint slides and notes given to us but I could revisit the videos and that really helped me to understand certain topics better. There are times that after the normal class session I sit to learn but I realize I do not understand one thing or the other. When that happens, I go back to the videos and this helps me a lot. (Male student, 23 years)

Another student indicated how videos made the learning process easier.

"It [videos] made learning easy. It made it fun and much easier. Sometimes when I am in class I may be tired or cannot concentrate but with the video, I can watch at my own time." (Male student, 21 years)

On the other hand, there were some perceived negative outcomes concerning the use of videos. Half of the students noted that the videos they watched for the Research Methods course made them lazy or reluctant. This perceived negative outcome was as a result of students forgoing the traditional class period because they had the videos to rely on. Some of the students stated this:

"...because it is something I watch at my leisure time, there is a tendency to be lazy. If I was going for a lecture, I know I have to be there but with the video because I watch at my own pace I can actually get lazy doing that." (Female student, 21 years)

Two of the students stated that there was no perceived learning outcome. One student noted this:

"It [videos] didn't have any great impact on my learning. It was normal."

(Female student, 22 years)

\section{Attitudes-learning approach}

Students' attitudes towards video based teaching were assessed by asking them if videos changed their learning approach in any way, as compared to non-video lectures or the traditional classroom teaching. Students' responses on their attitudes towards videos were generally positive. The responses made it clear that their attitudes to video were shaped by the perceived relevance to the lecture and course content, and their understanding of how the videos added value to other content, rather than replacing content.

Students indicated that videos changed their learning approach by making the entire learning process 'convenient' and 'comfortable'.

"I can wake up at any time and watch the videos. I can also watch them on the go like if I am in a bus, and this enables me grasp things perfectly. In non-video lectures, if you miss a topic or lecture or you are distracted 
momentarily in class, you may not get the opportunity to get what you have missed. The video lectures really helps me to recap what has been taught." (Male student, 23 years)

Other students also indicated that videos made them study more than they would have and also served as a point of reference. Almost a third of the students also indicated that videos made it easy for them to recall topics discussed in the classroom. Some students stated this:

"It [videos] made me learn more without noticing because I like watching. So instead of going to a group discussion, I would rather watch the videos because I will get things or topics better clarified." (Female student, 23 years)

"With videos I am always able to refer to something I did not get correctly in class. I cannot have that advantage with the traditional classroom lectures." (Female student, 21 years)

"...with the videos I am able to watch everything and then make my own notes later. Instead of trying to get everything understood or written in the classroom, videos made it easy for me to absorb whatever was taught." (Female student, 21 years)

However, four students suggested that videos did not change their attitude towards learning. It had no change on their learning approach. One student noted this:

"It [videos] didn't really change much [my learning approach] because after watching the videos I still went back to my notes to learn. Maybe it is because I didn't watch the full length of the videos so I had to come back to my books." (Female student, 21 years)

\section{Discussion}

This exploratory study examined the perceptions and attitudes of students concerning the use of videos as a medium of teaching and learning. We found that, students perceived videos in general as being of some benefit to their learning process. Overall, comments on videos as a medium of teaching were positive. Most of the respondents alluded to the fact that they had watched videos on the internet (particularly on YouTube) previously, to better understand a concept or topic taught in the classroom. This suggests that videos being part of a student's learning is widely accepted among the participants. We interpret this as an encouraging sign to establish videos as an integral part of the entire teaching and learning process. However, a student who is faced with circumstances (such as not having a laptop or any portable device) may be at a disadvantage, as compared to students whose circumstances are more favourable. We suggest that there should be a location where videos are made available on computers so students without such devices can have access to them.

It is worth noting that students had negative descriptions about the nature of the videos they watched in relation to their Research Methods course. Almost all the participants indicated that they had one issue or the other with the content and the 
format of all the videos they watched. With regard to the content, the students' comments suggest that the videos should have a different or modified approach than what they experienced. We suggest that to improve the content of the videos, it should depict authentic human behavior rather than scripted behaviour. The videos should be interactive and lively in nature. Also, we suggest that to make the content of these videos different from the traditional classroom period, there should be practice sessions in the videos which should be followed up with a thorough discussion during the actual class period.

Additionally, even though the participants indicated that the quality of the video was high, they also alluded that the size was too large. Almost all the participants suggested that the videos were too large in size. The large size can be attributed to the (high) quality of the videos. It may be that the students are oblivious of this fact, and may not want to give up the quality of the video for a reduced video size if they are aware. But, majority of the participants perceived that the videos they watched enhanced their learning outcomes. This implies that the students benefited from the videos they watched in relation to the Research Methods course. It suggests that videos are not only an important compliment to traditional classroom teaching, but are actually a means to achieving desired learning goals. The negative perceived outcome of videos can be overcome if the videos could be structured in a way that will also make it a requirement to attend the usual class session. This will eliminate the tendency for students to purposively or intentionally miss class. Also, the fact that most students' learning approach was enhanced by videos, reinforces the fact that videos are now very critical in the whole learning process. The main advantage for students with regard to their learning approach was that videos greatly assisted them to be more detailed in a convenient way.

\section{Conclusion}

The findings show that videos are very important to the participants' learning outcome and approach. However, it must also be noted that effectively using video requires effort and planning on the part of instructors. To make videos as a medium of instruction more effective than its current structure at the University of Ghana, we suggest that course instructors should explain the use of videos during the course (situating it in the course outline), stating how videos are planned to be used in the course, the relationship of the videos to course learning outcomes and assignments, as well as potential learning benefits. Course instructors must also facilitate discussions or clarifications based on the videos and most importantly, ensure that students are able to share their perceptions of the video over the period of the course (Fee and Budde-Sung 2014). Furthermore, videos should be considered as one component in a complex classroom activity system; such that learning outcomes of students and instructors are dependent on the manner in which videos are used as part of the overall academic environment (Karppinen 2005).

This study is one of the first articles to explore in detail, students' perceptions and attitudes towards video based teaching in Ghana, and provides interesting insights with regard to the concept and its application in tertiary institutions. 


\section{Limitations}

The study findings cannot be used to generalize for all students at the University of Ghana. The essence of this study was to capture the different representations of students with regard to the use of videos in combination with classroom teaching in a Research Methods course. Future studies could use the findings in this study as a basis to delve deeper into video based e-learning in other academic courses. Despite the limitations stated, we believe that this study makes a valuable contribution to the existing literature.

\section{Competing interests}

The authors declare that they have no competing interests.

Received: 20 January 2016 Accepted: 6 May 2016

Published online: 11 May 2016

\section{References}

M Alavi, Y Yoo, DR Vogel, Using information technology to add value to management education. Acad. Manag. J. 40(6), 1310-1333 (1997)

IT Awidi, Developing an e-learning strategy for public universities in Ghana. Educ. Q. 2, 66-69 (2008)

P Baggett, Role of temporal overlap of visual and auditory material in forming dual media associations. J. Educ. Psychol. 76(3), 408-417 (1984)

T Boyle, Design for multimedia learning (Prentice Hall, Upper Saddle River, NJ, 1997)

JS Brown, A Collins, P Duguid, Situated cognition and the culture of learning. Educ. Res. 18(1), 32-42 (1989)

KS Cennamo, Learning from video: factors influencing learners' preconceptions and invested mental effort. Educ. Technol. Res. Dev. 41(3), 33-45 (1993)

HJ Choi, SD Johnson, The effect of problem-based video instruction on learner satisfaction, comprehension and retention in college courses. Br. J. Educ. Technol. 38(5), 885-895 (2007)

RE Clark, Media and method. Educ. Technol. Res. Dev. 42(3), 7-10 (1994)

R Corston, AM Colman, Gender and social facilitation effects on computer competence and attitudes toward computers. J. Educ. Comput. Res. 14(2), 171-183 (1996)

CJ Dede, The evolution of distance learning: technology-mediated interactive learning. J. Res. Comput. Educ. 22(1), 247-264 (1990)

TD Duffy, DJ Cunningham, Constructivism: implications for the design and delivery of instruction, in Handbook of research for educational communications and technology: a project of the association for educational communications and technology, ed. by D.H. Jonassen (Macmillan, New York, 1996), pp. 55-85

A Fee, AEK Budde-Sung, Using video effectively in diverse classes: what students want. J. Manag. Educ. 38(6), 843-874 (2014)

A Fern, R Givan, JM Siskind, Specific-to-general learning for temporal events with application to learning event definitions from video. J. Artif. Intell. Res. 17, 379-449 (2011)

R Goldman, Video representations and the perceptivity framework: epistemology, ethnography, evaluation, and ethnics, in Video research in the learning sciences, ed. by R. Goldman, R. Pea, B. Barron, S.J. Derry (Lawrence Erlbaum Associates, London, 2007)

P Hakkarainen, T Saarelainen, H Ruokamo, Towards meaningful learning through digital video supported, case based teaching. Australas. J. Educ. Technol. 23(1), 87-109 (2007)

DH Jonassen, Computers as mind tools for schools. Engaging critical thinking (Prentice-Hall, Upper Saddle River, NJ, 2000)

P Karppinen, Meaningful learning with digital and online videos: theoretical perspectives. AACE J. 13(3), $233-250$ (2005)

RB Kozma, Learning with media. Rev. Educ. Res. 61(2), 179-211 (1991)

PG Lange, Publicly private and privately public: social networking on YouTube. J. Comput.-Mediat. Commun. 13(1), 361-380 (2008)

CA Lengnick-Hall, MM Sanders, Designing effective learning systems for management education: student roles, requisite variety, and practicing what we teach. Acad. Manag. J. 40(6), 1334-1368 (1997)

R Luppicini, Categories of virtual learning communities for educational design. Q. Rev. Dist. Educ. 4(4), 409-419 (2003)

BK Lynn, Teaching and learning in the digital world: Possibilities and challenges. LEARNing Landscapes. 6(2), 423 (2013)

RE Mayer, Applying the science of learning: evidence-based principles for the design of multimedia instruction. Am. Psychol. 63, 760-769 (2008)

RE Mayer, Multimedia learning, 2nd edn. (Cambridge University Press, New York, NY, 2009)

GC Nugent, Pictures, audio, and print: symbolic representation and effect on learning. Educ. Comm. Tech. J. 30(3), 163-174 (1982)

J O'Flaherty, C Phillips, The use of flipped classrooms in higher education: a scoping review. Internet High. Educ. 25, 85-95 (2015)

MQ Patton, Qualitative research and evaluation methods, 3rd edn. (Sage, Thousand Oaks, CA, 2002)

M Prensky, Listen to the natives. Educ. Leadersh. 63, 8-13 (2005)

D Rahm, BJ Reed, Going remote: the use of distance learning, the World Wide Web, and the Internet in graduate programs of public affairs and administration. Publ. Prod. Manag. Rev. 20(4), 459-471 (1997)

S Saltmarsh, T Swirski, "Pawns and prawns": international academics' observations on their transition to working in an Australian university. J. High. Educ. Pol. Manag. 32, 291-301 (2010)

D Silverman, A Marvasti, Doing qualitative research: a comprehensive guide (Sage, Thousand Oaks, CA, 2008) 
JB South, B Gabbitas, PF Merrill, Designing video narratives to contextualize content for ESL learners: a design process case study. Interact. Learn. Environ. 16(3), 231-243 (2008)

SG Strauss, Getting a clue: communication media and information distribution effects on group process and performance. Small Group Res. 27(1), 115-142 (1996)

MR Syed, Diminishing the distance in distance education. IEEE Multimedia 8(3), 18-21 (2001)

TL-P Tang, MJ Austin, Students' perceptions of teaching technologies, application of technologies, and academic performance. Comput. Educ. 53, 1241-1255 (2009)

RE Yellen, MA Winniford, CC Sanford, Extroversion and introversion in electronically-supported meetings. Inform. Manag. 28, 63-74 (1995)

\section{Submit your manuscript to a SpringerOpen ${ }^{\circ}$ journal and benefit from:}

- Convenient online submission

- Rigorous peer review

- Immediate publication on acceptance

- Open access: articles freely available online

- High visibility within the field

- Retaining the copyright to your article

Submit your next manuscript at $>$ springeropen.com 